\title{
Edge-on disk galaxies in the SDSS DR6: Fractions of bulgeless and other disk galaxies
}

\author{
S.J. Kautsch ${ }^{\star}$ \\ Department of Astronomy, University of Florida, 211 Bryant Space Science Center, Gainesville, FL 32611-2055, USA
}

Received 2008 Sep 29, accepted 2008 Nov 17

Published online 2008 Dec 28

Key words galaxies: fundamental parameters - galaxies: irregular - galaxies: spiral - galaxies: statistics - galaxies: structure

The aim of this study is to determine the fractions of different spiral galaxy types, especially bulgeless disks, from a complete and homogeneous sample of 15127 edge-on disk galaxies extracted from the sixth data release from the Sloan Digital Sky Survey. The sample is divided in broad morphological classes and sub types consisting of galaxies with bulges, intermediate types and galaxies which appear bulgeless. A small fraction of disky irregulars is also detected. The morphological separation is based on automated classification criteria which resemble the bulge sizes and the flatness of the disks. Each of these broad classes contains about $1 / 3$ of the total sample. Using strict criteria for selecting pure bulgeless galaxies leads to a fraction of $15 \%$ of simple disk galaxies. We compare this fraction to other galaxy catalogs and find an excellent agreement of the observed frequency of bulgeless galaxies. Although the fraction of simple disk galaxies in this study does not represent a "cosmic" fraction of bulgeless galaxies, it shows that the relative abundance of pure disks is comparable to other studies and offers a profound value of the frequency of simple disks in the local Universe. This fraction of simple disks emphasizes the challenge for formation and evolution models of disk galaxies since these models are hard pressed to explain the observed frequency of these objects.

(C) 2009 WILEY-VCH Verlag GmbH \& Co. KGaA, Weinheim

\section{Introduction}

Simple disk galaxies are flat, late-type disk galaxies of the morphological Hubble class $\sim \mathrm{Sd}$ and later without a bulge component (e.g., Goad \& Roberts 1979, 1980; Karachentsev 1989; Karachentsev et al. 1992; Kautsch et al. 2005b). The formation and evolution of such thin galaxies is not yet well understood in the framework of $\Lambda$ cold dark matter models. Simulations have difficulties in producing diskdominated and bulgeless galaxies. The simulated disks are smaller, denser and have lower angular momentum than observed, known as the angular momentum problem. Adding feedback processes to the simulations can improve the creation of disk galaxies to some extent (e.g., Okamoto et al. 2005; Scannapieco et al. 2008), but D'Onghia \& Burkert (2004), D'Onghia et al. (2006), Köckert \& Steinmetz (2007) and Piontek \& Steinmetz (2008) show that producing the observed structural and kinematical properties of disk-dominated galaxies and simple disks cannot be improved by adding feedback processes and by increasing the numerical resolution of the simulations. Therefore, the formation mechanisms of simple disks remain enigmatic.

During and after their formation, disk-dominated galaxies and simple disks are very sensitive to various processes that are responsible for transforming those objects into bulge-dominated galaxies or even destroy the disks. The majority of disk-dominated and simple disk galaxies

^ Corresponding author: stefan@astro.ufl.edu are located in group and low-density environment (Kautsch, Gallagher \& Grebel 2008). Hence, this type of environment should preserve the bulgeless shape of those galaxies. The contrary is observed as mergers are the dominant interaction process in groups (Barnes 1985) and the group environment is effective in transforming morphologies (Kautsch et al. 2008b; Tran et al. 2008). Minor mergers already lead to bulge growth (e.g., D'Onghia et al. 2006; Cox et al. 2008b; Kazantzidis et al. 2008) and the heating of the thin, stellar disk (Purcell, Kazantzidis \& Bullock 2008). Near equal mass mergers can take place across all different galaxy environments where disk-dominated and simple disk galaxies reside (Karachentsev, Karachentseva \& Parnovskij 1993; Kautsch, Grebel \& Gallagher 2005) and affect the overwhelming majority of Milky Way-sized halos as shown in merger-tree statistics within $\Lambda$ CDM N-body simulations (Stewart et al. 2008). It is assumed that $\sim 70 \%$ of these Milky Way-sized halos contain disk-dominated galaxies and $\sim 11 \%$ of the halos are the hosts of simple disks (Stewart et al. 2007, and referenced therein). Undergoing such a merger event is basically infaust for a disk-dominated galaxy and especially simple disks, i.e., N-body simulations of mergers exhibit that the stellar disk can be - but is not always (Koda, Milosavljevic \& Shapiro 2007; Hopkins et al. 2008) - completely disrupted and morphologically transformed into an early-type galaxy (e.g., Toomre 1977; Steinmetz 2003; Cox \& Loeb 2008).

So called "pseudobulges" can grow due to internal disk instabilities. In this secular evolution model gas sinks into 
the disk center and the stars from a subsequent central star-formation period form a bulge (Kormendy \& Kennicutt 2004; Kormendy \& Fisher 2005). Galactic bars support the gas flow towards the galactic centers and thus are important for secular evolution. Bars are frequently detected in bulgeless galaxies (Barazza, Jogee \& Marinova 2008), making simple disk galaxies potential candidates for ongoing secular evolution.

The first comprehensive catalog of edge-on diskdominated galaxies is the "Flat Galaxy Catalog" (FGC, Karachentsev et al. 1993) and its extension, the "Revised Flat Galaxy Catalog" (RFGC, Karachentsev et al. 1999). FGC and RFGC are optical all-sky surveys. RFGC contains 4236 visually selected "flat" galaxies. A collection of disk-dominated galaxies in the near-infrared is gathered in "The 2MASS-selected Flat Galaxy Catalog" (Mitronova et al. 2004).

In order to contribute to a better characterization of flat galaxies, Kautsch et al. (2005b; 2006a) carried out a work which compiled a uniform sample of disk-dominated galaxies in the optical wavelengths from the Sloan Digital Sky Survey (SDSS, York et al. 2000). The SDSS is ideal for the identification of such galaxies with its deep, multi-wavelength, homogeneous and large-area coverage. They analyzed SDSS data from the Data Release 1 (DR1, Abazajian et al. 2003) and compiled a catalog of 3169 galaxies with prominent edge-on stellar disks (Kautsch et al. 2006a, hereafter "the catalog," which is accessible online1 and Kautsch et al. 2006b). Using an automated algorithm, galaxies in the catalog are divided into galaxies with bulges, intermediate types and simple disk galaxies and subclasses. $15.8 \%$ of the catalog galaxies are found to be simple disks. This demonstrates that bulgeless galaxies are frequent, especially among intermediate-mass star-forming galaxies (Matthews \& Gallagher 1997). This frequency increases up to $1 / 3$ of the catalog galaxies when puffy disks are included. The boundaries between the galaxy types in the catalog are not sharp, suggesting simple disks are the faint end in a continuum of disk galaxy properties, e.g., surface brightness.

Since DR1, SDSS has undergone significant changes. DR1 provides a survey area of $2099 \mathrm{deg}^{2}$ of imaging data. The data releases DR2 (Abazajian et al. 2004), DR3 (Abazajian et al. 2005), DR4 (Adelman-McCarthy et al. 2006), DR5 (Adelman-McCarthy et al. 2007) and DR6 (Adelman-McCarthy et al. 2008) are now available. DR6 covers $9583 \mathrm{deg}^{2}$ of imaging in total and has an $r$-band depth of approximately 22.2 mag. Hence, DR6 contains more than four times the footprint area of DR1. In addition, changes were made between DR1 and DR2 concerning the deblending of overlapping objects. This correction should improve the detection of individual galaxies.

In this study we collect edge-on disk galaxies as done in the catalog (Kautsch et al. 2006a) but use the larger and newer database of the SDSS DR6 and we apply improved

\footnotetext{
${ }^{1}$ http://vizier.cfa.harvard.edu/viz-bin/VizieR?-source=J/A+A/445/765
}

morphological separation criteria. This allows us to study the fractions of different edge-on disks in a more robust statistical manner. The galaxies in this new sample are also divided into morphological classes using a new approach compared to the catalog. The improved object detection and especially the much larger coverage area in the sky should provide updated statistics, accuracy and homogeneity of the fractions of disk-dominated galaxies in the local Universe. In addition, we provide a unique comparison of fractions of bulgeless disk galaxies from several catalogs and derive a robust estimation of the frequency of simple disks among spirals in the local Universe. The knowledge of this fraction is crucial for studies about the formation, evolution and survival of disk-dominated galaxies. Therefore, this work delivers useful statistical results for several follow-up studies such as the fraction of simple disk galaxies at high redshifts.

This article has the following structure: in Sect. 2 we describe how edge-on disk galaxies were selected in previous works and in this present study. The quantified morphological classification of galaxies with and without bulges is discussed in Sect. 3. The results of this study in terms of numbers and fractions and a comparison to the fractions of recently published studies are shown in Sect. 4. In Sect. 5 we summarize the results.

\section{Data acquisition}

In the catalog, edge-on disk galaxies were selected based on selection criteria that are similar to those of the original approach by Karachentsev et al.'s catalogs FGC and RFGC. The original object selection by Karachentsev et al. $(1993 ; 1999)$ is based on the visual identification of galaxies with an axial ratio $a / b \geq 7$ and a major axis diameter of $\gtrsim 40^{\prime \prime}$ in the blue band POSS- 1 copies and ESO/SERC photographic plates. In order to come as close as possible to these original values, a training set of RFGC galaxies that were recovered in the SDSS DR1 was analyzed in the cata$\log$ with respect to their sizes, magnitudes and color distribution. This allowed Kautsch et al. (2006a) to translate the original object selection from the RFGC into a query that selects all galaxies brighter than $m_{g}=20$ from the DR1 "Best Galaxy Table" with axial ratios $>3$ and a major axis diameter $>30^{\prime \prime}$ within certain broad color limits. For the details of this query, please consult Kautsch et al. (2006a; 2006b). Our intention now is to collect edge-on galaxies from the DR6 in the same reproducible fashion. Therefore we apply the SDSS CasJobs on the SDSS Context DR6 to the "Galaxy Table". CasJobs 1 is an online interface that performs queries on various SDSS datasets using the "Structured Query Language" (SQL) and the "Galaxy Table" is the table of the SDSS data archive that contains all parameters for objects selected as galaxies in DR6 with the highest quality at the time of the data release. The query used in the catalog and in this study selects all galaxies with: i) an axial ratio $a / b>3$

\footnotetext{
2 http://casjobs.sdss.org/CasJobs
} 
( $a$ major axis, $b$ minor axis) in the $g$-band, ii) an angular major axis diameter $a>30^{\prime \prime}$ in the $g$-band, iii) colors in the range of $-0.5<g-r<2$ and $-0.5<r-i<2$, iv) a magnitude limit in the $g$-band $<20$ mag. These selection criteria have the following form in SQL:

\section{SELECT*}

into mydb.edge_on_dr6_catalog from

Galaxy as G

WHERE G.petroMag_g $<20$

and (G.isoA_g/G.isoB_g) $>3$

and G.isoA_g $>37.8 \mathrm{pixel}$ this corresponds to an angular radius of $15^{\prime \prime}$

and (G.dered_g - G.dered_r) between -0.5 and 2

and (G.dered_r - G.dered_i) between -0.5 and 2

This query leads to a sample of 27308 objects. A visual inspection shows a non-negligible number of contaminants. The contaminants are mostly stellar refraction spikes and artifacts such as satellite/meteor tracks, empty images as well as face-on galaxies where elongated structures (e.g., bars, spiral arms) simulate an edge-on disk appearance. In addition, some of the galaxies are also affected by "shredding" (Abazajian et al. 2004). This means that a single object has more than one unique detection and therefore multiple entries in the SDSS database. In the catalog, these contaminants were removed manually. Because of the large numbers in the present sample, this approach is inefficient and should be quantified. We find that the majority of the contaminants are automatically removed by excluding the objects flagged with the following DR6 PhotoFlags:

- "edge" indicates galaxies truncated on the survey borders;

- "saturated" indicates saturated pixels;

- "notchecked" indicates that SDSS deblending may be unreliable;

- "too_few_good_detections" indicates objects with no good centroid found in all bands;

- "petroMagErr" $>0.3$ in $g, r, i$ indicates the magnitude errors.

This leads to a DR6 sample of 15176 objects. We exclude also the objects that do not have Petrosian radii,

"petroR50_r" and "petroR90_r." The final DR6 edgeon galaxy sample contains 15127 objects.

We select various large random samples in order to estimate the remaining contribution of contaminants in this final sample. We find a rate of $5 \%$ of false detections for the whole sample, mainly due to shredded galaxies, elongated HII regions in disks, projected objects in spiral arms, empty images, projected objects in halos of saturated stars and rarely, stellar spikes that are still present in the sample.

3 These flags are associated with every unique object in the database and contain important information of the quality of the object: http://www.sdss.org/dr6/products/catalogs/flags.html

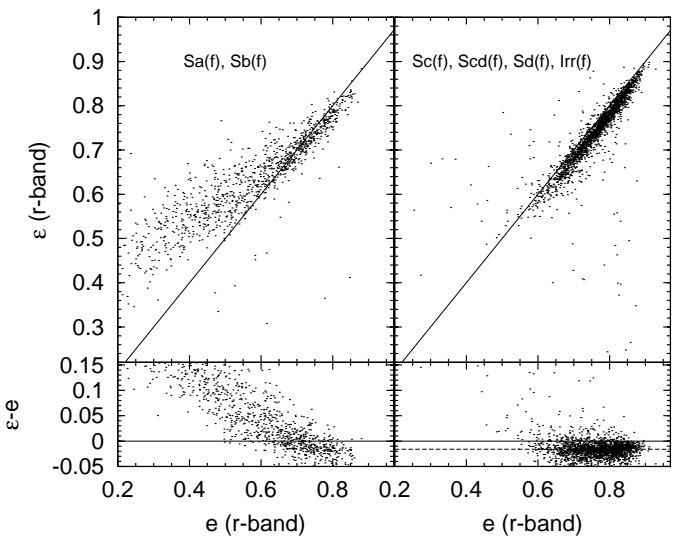

Fig. 1 This figure shows the relation between the luminosity weighted mean ellipticity $\varepsilon$ (ordinate) and the adaptive ellipticity e (abscissa) for all galaxies in the catalog in the SDSS $r$-band. Early-type disk galaxies are plotted in the left part and late-type disks in the right part of this figure. The scatter plots are presented in the lower section of the figure. The fine solid lines in the upper diagrams indicate the linear relation with a slope of one. The solid lines in the bottom diagrams show the location of $\varepsilon-\mathrm{e}=0$. The dashed line in the scatter plot for the late disks (bottom right) indicates the derived mean offset between $\varepsilon$ and e.

\section{Morphological separation}

In the catalog we used an automated morphological classification in order to define six types of edge-on disk galaxies. These are galaxies with bulges ( $\mathrm{Sa}(\mathrm{f}), \mathrm{Sb}(\mathrm{f}))$; simple disk galaxies $(\operatorname{Sd}(\mathrm{f})$ ); and disk-dominated intermediate types $(\mathrm{Sc}(\mathrm{f}), \operatorname{Scd}(\mathrm{f}))$ as well as disky edge-on irregulars (Irr(f)). We follow the terminology by de Vaucouleurs (1959) where spiral galaxies are flagged with a letter associated with the shape of the spiral arms (ring-shaped galaxies have an "r" and s-shaped galaxies have an "s"). We instead are using an " $\mathrm{f}$ " in brackets in order to indicate that the galaxies contain flat disks seen edge on.

The morphological separation in the catalog is based on the concentration index (CI) and the luminosity-weighted mean ellipticity of the elliptical isophotes $(\varepsilon)$, both in the SDSS $r$-band. The CI is a measure of the bulge size for edge-on galaxies and is defined as the ratio of the Petrosian radii that contain $90 \%$ and $50 \%$ of the Petrosian flux in the same band in a circular aperture. We used the Petrosian radii that are listed in the "Galaxy Table." The CI separation values divide the early $(\mathrm{Sa}(\mathrm{f}), \mathrm{Sb}(\mathrm{f}))$ from the late types $(\mathrm{Sc}(\mathrm{f})$, $\operatorname{Scd}(f), \operatorname{Sd}(f))$. In addition, low CI values separate the $\operatorname{Irr}(f)$ class from the late types. We use the same CI separation criteria as in the catalog because the Petrosian radii did not undergo changes between DR1 and DR6.

$\varepsilon$ is a discriminator of the flatness of edge-on disks and was measured directly on the individual galaxy images of the galaxies in the catalog. This measurement was performed with the MIDAS surfphot package. We fit elliptical 
isophotes to the galaxies and derived $\varepsilon$ as the weighted mean ellipticity of all isophote levels of an individual galaxy.

We replace the method finding $\varepsilon$ by using variables offered directly from the SDSS tables. The new method offers consistency within the SDSS parameters. In addition, with this method it is simple to reproduce our morphological separation criteria in a fast and efficient way. Disk flatness can be expressed through the adaptive moments provided in the DR6 "Galaxy Table." The moments are measured from the ellipticity and size of the objects within the SDSS pipelinet. The adaptive second moments from the SDSS (mE1 and $\mathrm{mE} 2$ ),

$e_{+}=\mathrm{mE} 1$,

$e_{\times}=\mathrm{mE} 2$,

can be converted into an "adaptive" ellipticity (e), using $a$ and $b$ as major and minor axis, respectively (c.f., Vincent $\&$ Ryden 2005):

$\mathrm{e}=1-\frac{b}{a}=1-\sqrt{\frac{1-\sqrt{e_{+}^{2}+e_{\times}^{2}}}{1+\sqrt{e_{+}^{2}+e_{\times}^{2}}}}$.

We now compare $\varepsilon$ with e for all galaxies from the catalog. The upper part of Fig. 1 1 shows the relation between $\varepsilon$ and e for the early-disk types (left) and the late types (right). The diagrams on the bottom of Fig. 11 show the scatter plots. Early disks ( $\mathrm{Sa}(\mathrm{f}), \mathrm{Sb}(\mathrm{f})$ ) (on the left side of this plot) tend to have a rounder shape (i.e., lower ellipticity values) of e compared with $\varepsilon$. In contrast, late disk types $\operatorname{Sc}(\mathrm{f}), \operatorname{Scd}(\mathrm{f})$, $\operatorname{Sd}(f)$ and $\operatorname{Irr}(\mathrm{f})$ (shown in the right part of the figure) are flatter using e instead of $\varepsilon$. We also see some late-type outliers having significantly lower $\varepsilon$ compared to e. A visual inspection of the outliers exhibits that many of these points belong to misclassified $\operatorname{Irr}(\mathrm{f})$ types in the catalog.

The limiting values of $\varepsilon$ in table 1 in the catalog must be recalculated in order to separate the galaxies into different morphologies based on e. The late types are constantly shifted from a simple linear relation with a slope of one between $\varepsilon$ and e as shown in the right bottom part of Fig. 1. We derive a mean offset of $\varepsilon-\mathrm{e}=-0.016$, indicated as dashed line in Fig. 1. Because the late disks are flatter in e, the offset must be added to $\varepsilon$ in order to resemble the limiting values of the catalog. We emphasize that the main intention of this investigation is to study the fractions of disk-dominated galaxies. Therefore, early-type disks (classified as $\mathrm{Sa}(\mathrm{f})$ and $\mathrm{Sb}(\mathrm{f})$ ) are less important for this purpose. However, we use a histogram of the number distribution of e for the galaxies classified as $\mathrm{Sa}(\mathrm{f})$ and $\mathrm{Sb}(\mathrm{f})$ in the catalog. With the aid of this histogram (Fig. 2) we define a value of $\mathrm{e}=0.4$ as the best separation value that resembles the dividing limit between $\mathrm{Sa}(\mathrm{f})$ and $\mathrm{Sb}(\mathrm{f})$. The limiting values for $\mathrm{CI}$ and e are collected in Table 1

4 http://www.sdss.org/dr6/algorithms/adaptive.html

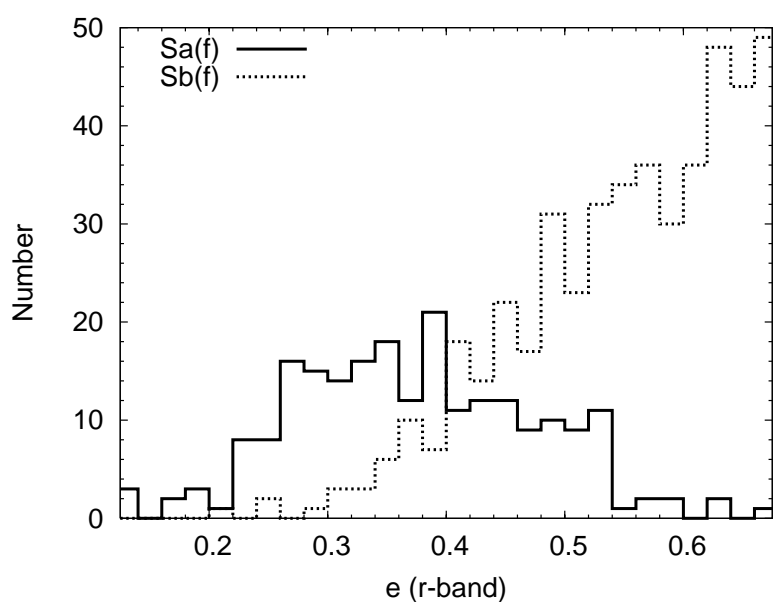

Fig. 2 The number distribution for the early-type disk galaxies of the adaptive ellipticity (e) is shown in this histogram. $\mathrm{Sa}(\mathrm{f})$ galaxies, the class containing galaxies with the largest bulges, are drawn with a solid line. $\mathrm{Sb}(\mathrm{f})$ galaxies are indicated with a dotted line.

Table 1 The limiting values of the adaptive ellipticity (e) and concentration index (CI) between the morphological classes are listed in this table. The values are valid for the SDSS $r$-band. In general, note that the galaxies near boundaries have the least certain classification.

\begin{tabular}{lrrrr}
\hline Class & \multicolumn{2}{c}{$\mathrm{e}$} & \multicolumn{2}{c}{$\mathrm{CI}$} \\
\hline & Lower Limit & Upper Limit & Lower Limit & Upper Limit \\
\hline $\mathrm{Sa}(\mathrm{f})$ & -- & $<0.40$ & $\geq 2.70$ & -- \\
$\mathrm{Sb}(\mathrm{f})$ & $\geq 0.40$ & -- & $\geq 2.70$ & -- \\
$\mathrm{Sc}(\mathrm{f})$ & -- & $<0.766$ & $\geq 2.15$ & $<2.70$ \\
$\mathrm{Scd}(\mathrm{f})$ & $\geq 0.766$ & $<0.816$ & $\geq 2.15$ & $<2.70$ \\
$\mathrm{Sd}(\mathrm{f})$ & $\geq 0.816$ & -- & -- & $<2.70$ \\
$\mathrm{Irr}(\mathrm{f})$ & -- & $<0.816$ & -- & $<2.15$ \\
\hline
\end{tabular}

\section{Results}

\subsection{Fractions of different types of edge-on galaxies}

We apply the new limiting values from Table 1 to the new DR6 edge-on galaxy sample. Figures 3, 4, 5, 6, 7, and 8 show example images of galaxies with types $\mathrm{Sa}(\mathrm{f}), \mathrm{Sb}(\mathrm{f})$, $\operatorname{Sc}(f), \operatorname{Scd}(f), \operatorname{Sd}(f)$ and $\operatorname{Irr}(f)$, respectively. These images have an angular size of 100 square arcsec and north is to the top, east to the left. The images are downloaded from the SDSS Image List Too 5. The number results are shown in Table 2. In this table we also reprint the numbers of the classes found in the catalog on the right side. The largest difference between the catalog and this study is the fraction of $\operatorname{Irr}(f)$. Their increased number in the present study is due to the contamination of misclassified objects, as revealed in a visual inspection of randomly selected $\operatorname{Irr}(f)$ types. Aside from this, the fractions of the different types are almost the

\footnotetext{
5 http://cas.sdss.org/dr6/en/tools/chart/list.asp
} 
Table 2 The galaxy classes and their fractions are shown in this table. The absolute numbers of galaxies in the main morphological classes (Col. 2) and their percentages (Col. 3) are listed in this table. For comparison the numbers and percentages of the catalog are given in Cols. 4 and 5 .

\begin{tabular}{lcccc}
\hline Database & \multicolumn{2}{c}{ SDSS DR6 } & \multicolumn{2}{c}{ SDSS DR1 } \\
\hline General Class & Number & Percentages & Number & Percentages \\
\hline Sa(f) & 966 & 6 & 222 & 7 \\
Sb(f) & 3993 & 26 & 843 & 26 \\
Sc(f) & 4835 & 32 & 1005 & 32 \\
Scd(f) & 2257 & 15 & 503 & 16 \\
Sd(f) & 2220 & 15 & 501 & 16 \\
Irr(f) & 856 & 6 & 95 & 3 \\
Total & 15127 & 100 & 3169 & 100 \\
\hline
\end{tabular}

same. For completeness considerations of the classes we refer to Sect. 6 in the catalog.

The limiting values for $\mathrm{Sd}(\mathrm{f})$ of the catalog are based on fairly conservative separation criteria in order to minimize possible contamination from other classes. So the fraction of simple disks is $15 \%$ in the present study. In the catalog we show that it is also possible to include the $\operatorname{Scd}(\mathrm{f})$ types in an extended bulgeless disk class. The fraction of this extended bulgeless disk class ( $\operatorname{Scd}(\mathrm{f})$ and $\operatorname{Sd}(\mathrm{f}))$ is $30 \%$ of the total DR6 sample. This value is similar to the value of $32 \%$ of the catalog. Therefore, the fraction of the extended bulgeless disk class in this work and the catalog corresponds to roughly $1 / 3$ of the total sample of edge-on disk galaxies. However, the contamination by other types is larger than with the more rigorous defined limits for simple disks.

\subsection{Comparison with other studies}

It is generally known that disk galaxies of late Hubble morphologies are often bulgeless (e.g., Hubble 1936; Böker et al. 2002; Kormendy \& Kennicutt 2004). However, accurate number statistics of the fraction of simple disk galaxies are rare, probably due to the lack of large samples.

Barazza et al. (2008) studied the fraction of bars in disk galaxies and they also analyzed the fraction of bulgeless galaxies based upon visual inspection. The fraction of bulgeless disks is $\sim 20 \%$ in their sample. The sample they used is selected from the SDSS with $-18.5 \leq M_{g}<-22$ and redshifts between $0.01<z<0.03$. Disk galaxies are selected using a color cut, and galaxies with inclinations larger than $60^{\circ}$ are omitted. Their fraction of bulgeless galaxies is close to that of the simple disks $(\operatorname{Sd}(\mathrm{f}))$ found here. The $\sim 5 \%$ difference can be explained by considering that Barazza et al. (2008) used a color cut for the disk galaxy selection. This could exclude red spiral types and therefore slightly offset their sample towards bluer and later disk types.

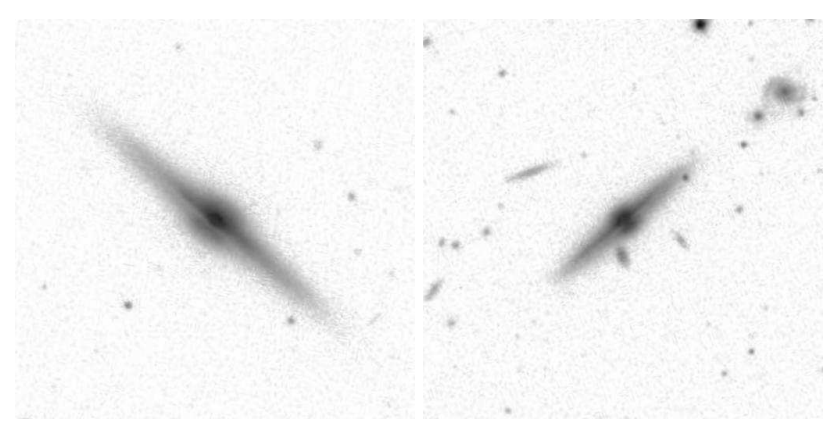

Fig. $3 \quad \mathrm{Sa}(\mathrm{f}): \quad \mathrm{J} 111146.36+364442.3$ (right panel), $\mathrm{J} 143650.68+294525.5$ (left panel).

Koda et al. (2007) used the "Tully Galaxy Catalog"6 in order to select galaxies with morphological information given in that catalog. The fraction of $\mathrm{Sd}$ galaxies is $11 \%$. Considering the restriction to local (dist $<20 \mathrm{~h}^{-1}$, $-17<M_{B}<-20$ ) galaxies, their fraction is similar to ours. The small difference of $\sim 4 \%$ is probably an effect of visual classification: The "Tully Galaxy Catalog" is not restricted to edge-on galaxies. Less inclined objects can exhibit central light concentrations from nuclei (e.g., Walcher et al. 2006) which are not seen edge-on. For this reason, some Sd would have been classified as earlier types.

In Allen et al. (2006), 10095 galaxies with $m_{B}<20$ from the "Millennium Galaxy Catalog" (Liske et al. 2003) were morphologically analyzed using two component Sersic spheroid + exponential disk decomposition (GIM2D, Simard et al. 2002, 2008). The fraction of pure exponential disks is $14 \%$ and comparable to the fraction of simple disks in our sample.

The Neighboring Galaxy Catalog (Karachentsev et al. 2004) contains 77 disk-like objects among 451 galaxies with a magnitude limit of $M_{B} \gtrsim-12$ and a distance $\leq 10$ Mpc. This study exhibits an Sd fraction of $21 \pm 5 \%$. This catalog includes low-luminosity galaxies and this increases the completeness of the fraction of simple disks at the faint end. Therefore, the simple disk fraction is larger compared to the DR6 sample.

By comparing the simple disk fractions of this study with its progenitor catalogs, the catalog (Kautsch et al. 2006a) (16\%) and the RFGC (Karachentsev et al. 1999) (17\%), also reveal an excellent agreement of the fractions.

This comparison shows a good agreement of the fractions of simple disk galaxies ( $\mathrm{Sd}$ types) between different studies which are using large numbers of analyzed galaxies. The results of this comparison are summarized in Table 3. Although these studies use different luminosity, distance, inclination and morphological selection criteria, the average fraction of simple disks is $16.2 \% \pm 3.2 \%$ among late-type galaxies.

\footnotetext{
${ }^{6}$ http://haydenplanetarium.org/universe/duguide/exgg_tully.php
} 


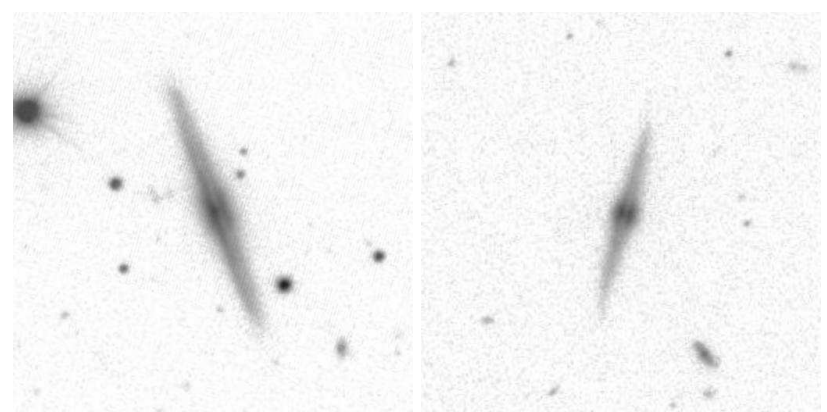

Fig. $4 \quad \mathrm{Sb}(\mathrm{f})$ : $\quad \mathrm{J} 172506.84+565241.8$ (right panel), J231006.72-093953.6 (left panel).

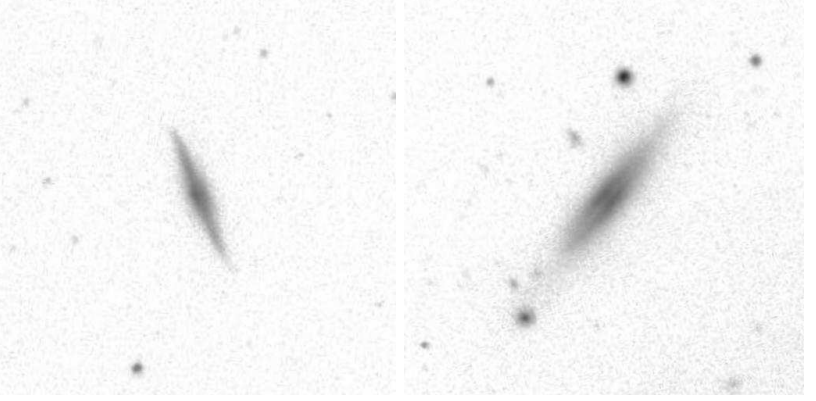

Fig. 5 Sc(f): J020800.74-082442.1 (right panel), $\mathrm{J} 143917.88+200439.7$ (left panel).

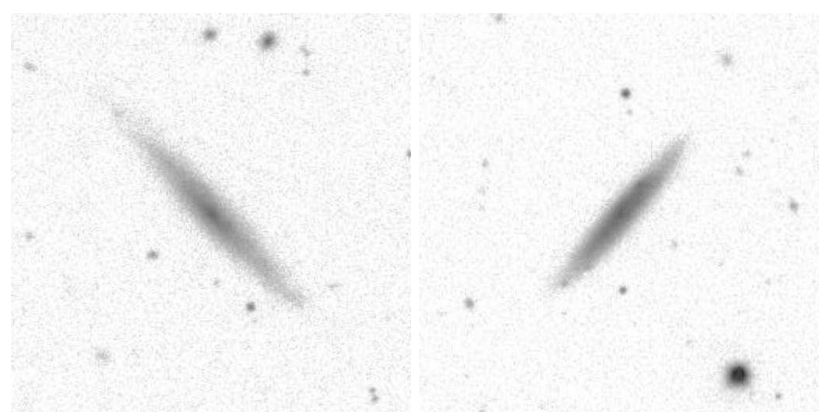

Fig. 6 Scd(f): J225828.27-103332 (right panel), $\mathrm{J} 124943.24+044610.1$ (left panel).

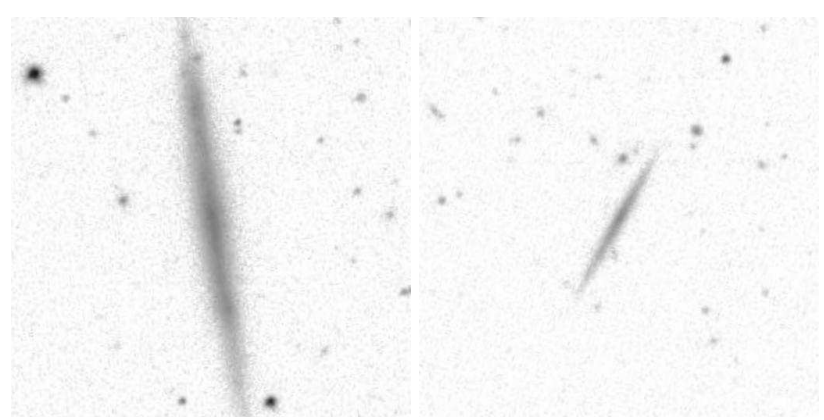

Fig. $7 \quad \mathrm{Sd}(\mathrm{f})$ : $\quad \mathrm{J} 214439.43-064122.5$ (right panel), $\mathrm{J} 104658.44+382728$ (left panel).

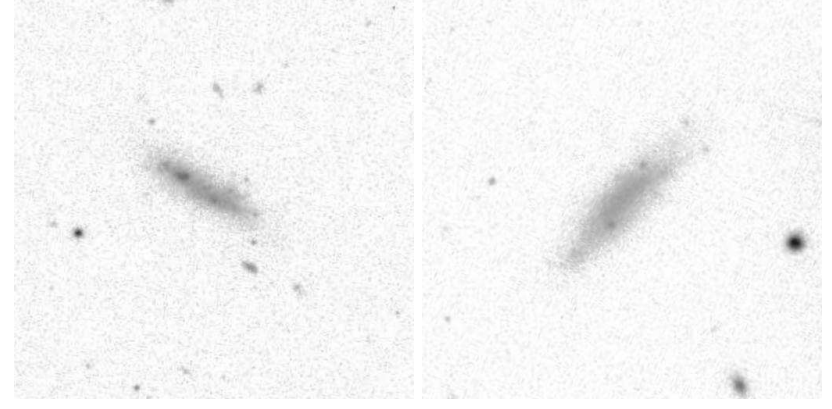

Fig. $8 \quad \operatorname{Irr}(\mathrm{f}): \quad \mathrm{J} 091028.24+071111.7$ (right panel), $\mathrm{J} 104210.63+632430.5$ (left panel).

Table 3 A comparison of the fractions of simple disk galaxies in the present work and other recent studies is shown in this table. The fractions (in percentages) are listed in Col. 1, and the method of the morphological classification in Col. 2.

\begin{tabular}{lcl}
\hline & Fraction [\%] & Method \\
\hline This study & 15 & Automated \\
Kautsch et al. 2006a & 16 & Automated \\
Barazza et al. 2008 & 20 & Visual \\
Koda et al. 2007 & 11 & Visual \\
Allen et al. 2006 & 14 & Automated \\
Karachentsev et al. 2004 & 21 & Visual \\
Karachentsev et al. 1999 & 17 & Visual \\
\hline
\end{tabular}

\section{Summary and discussion}

This study presents the fractions of disk-dominated galaxies and other edge-on disk galaxy types selected from the SDSS DR6. Using a quantitative method that measures the disk flatness and the bulge size, this sample is divided into morphological classes of galaxies with bulges, intermediate types and apparently bulgeless objects. Each of these broad types contains roughly $1 / 3$ of the total sample. Further subdivision is applied to these types and allows us to define the fraction of simple disk galaxies, $\operatorname{Sd}(\mathrm{f})$, to be $15 \%$ of the total disk galaxy sample. The fractions of other morphological Hubble types of disk galaxies are also presented and compared to the catalog of edge-on disk galaxies (Kautsch et al. 2006a).

We also present a comparison of the frequency of simple disk galaxies from various galaxy catalogs and find a simple disk fraction of $\sim 16 \% \pm 3 \%$ on average. The fractions are a robust result because of the excellent agreement between the studies, although various criteria were used to select the galaxies. Small differences of the $\mathrm{Sd}$ fractions can be explained qualitatively. We conclude that simple disks are a common galaxy type, although formation and evolution models are challenged by explaining bulgeless galaxies. Hence, a comprehensive description of simple disks remains to be explored.

Acknowledgements. The author expresses his gratitude to the anonymous referee and to Dr. Fabio D. Barazza, Prof. Anthony 
H. Gonzalez and Leah E. Simon for the support of this work and critical reading of the manuscript. The author also wants to thank Prof. Eva K. Grebel and Prof. Jay S. Gallagher for their kind support. Funding for the SDSS (http://www.sdss.org/) and SDSS-II has been provided by the Alfred P. Sloan Foundation, the Participating Institutions, NSF, the U.S. Department of Energy, NASA, the Japanese Monbukagakusho, and the Max Planck Society, and the Higher Education Funding Council for England. The SDSS is managed by the Astrophysical Research Consortium (ARC) for the Participating Institutions. The Participating Institutions are the American Museum of Natural History, AIP, Univ. of Basel, Univ. of Cambridge, Case Western Reserve Univ., The Univ. of Chicago, Drexel Univ., Fermilab, the Institute for Advanced Study, the Japan Participation Group, The Johns Hopkins Univ., the Joint Institute for Nuclear Astrophysics, the Kavli Institute for Particle Astrophysics and Cosmology, the Korean Scientist Group, the Chinese Academy of Sciences (LAMOST), Los Alamos National Lab., MPIA, MPA, New Mexico State Univ., Ohio State Univ., Univ. of Pittsburgh, Univ. of Portsmouth, Princeton Univ., USNO, and the Univ. of Washington.

\section{References}

Abazajian, K., Adelman-McCarthy, J.K., Agüeros, M.A., et al.: 2003, AJ 126, 2081

Abazajian, K., Adelman-McCarthy, J.K., Agüeros, M.A., et al.: 2004, AJ 128, 502

Abazajian, K., Adelman-McCarthy, J.K., Agüeros, M.A., et al.: 2005, AJ 129, 1755

Adelman-McCarthy, J.K., Agüeros, M.A., Allam, S.S., et al.: 2006, ApJS 162, 38

Adelman-McCarthy, J.K., Agüeros, M.A., Allam, S.S., et al.: 2007, ApJS 172, 634

Adelman-McCarthy, J.K., Agüeros, M.A., Allam, S.S., et al.: 2008, ApJS 175, 297

Allen, P.D., Driver, S.P., Graham, A.W., et al.: 2006, MNRAS 371, 2

Barazza, F.D., Jogee, S., Marinova, I.: 2008, ApJ 675, 1194

Barnes, J.: 1985, MNRAS 215, 517

Böker, T., Laine, S., van der Marel, R.P., et al.: 2002, AJ 123, 1389

Cox, T.J., Loeb, A.: 2008, MNRAS 386, 461

Cox, T.J., Jonsson, P., Somerville, R.S., Primack, J.R., Dekel, A.: 2008, MNRAS 384, 386

de Vaucouleurs, G.: 1959, Handbuch der Physik, Vol. 53, Astrophysik IV: Stellar Systems, Springer-Verlag, Berlin, Göttingen, Heidelberg

D’Onghia, E., Burkert, A.: 2004, ApJ 612, L13

D'Onghia, E., Burkert, A., Murante, G., Khochfar, S.: 2006, MNRAS 372, 1525

Fujita, Y.: 2004, PASJ 56, 29

Goad, J.W., Roberts, M.S.: 1979, BAAS 11, 668

Goad, J.W., Roberts, M.S.: 1981, ApJ 250, 79

Gunn, J.E., Gott, J.R., III: 1972, ApJ 176, 1

Heidmann, J., Heidmann, N., de Vaucouleurs, G.: 1972, MmRAS 75,85

Hopkins, P.F., Cox, T.J., Younger, J.D., Hernquist, L.: 2008, astro$\mathrm{ph} / 0806.1739$

Hubble, E.P.: 1936, Realm of the Nebulae, Yale University Press, New Haven

Karachentsev, I.: 1989, AJ 97, 1566

Karachentsev, I.D., Georgiev, Ts.B., Kajsin, S.S., Kopylov, A.I., et al.: 1992, A\&AT 2, 265
Karachentsev, I.D., Karachentseva, V.E., Parnovskij, S.L.: 1993, AN 314, 97 (FGC)

Karachentsev, I.D., Karachentseva, V.E., Kudrya, Yu.N., Sharina, M.E., Parnovsky, S.L.: 1999, Bull. Special Astrophys. Obs. 47, 5 (RFGC) 
Karachentsev, I.D., Karachentseva, V.E., Huchtmeier, W.K., Makarov, D.I.: 2004, AJ 127, 2031

Kautsch, S.J., Grebel, E.K., Gallagher, J.S., III: 2005, AN 326, 496

Kautsch, S.J., Grebel, E.K., Barazza, F.D., Gallagher, J.S., III: 2005b, AN 326, 595

Kautsch, S.J., Grebel, E.K., Barazza, F.D., Gallagher, J.S., III: 2006a, A\&A 445, 765 (the catalog)

Kautsch, S.J., Grebel, E.K., Barazza, F.D., Gallagher, J.S., III: 2006b, A\&A 451, 1171

Kautsch, S.J., Gallagher, J.S., III, Grebel, E.K.: 2008a, A\&A, submitted

Kautsch, S.J., Gonzalez, A.H., Soto, C.A., Tran, K.-V. H., Zaritsky, D., Moustakas, J.: 2008b, ApJ 688, L5

Kazantzidis, S., Bullock, J.S., Zentner, A.R., Kravtsov, A.V., Moustakas, L.A.: 2008, ApJ 688, 254

Köckert, F., Steinmetz, M.: 2007, in: F. Combes, J. Palous (eds.), Galaxy Evolution Across the Hubble Time, IAU Symp. 235, p. 114

Koda, J., Milosavljevic, M., Shapiro, P.R.: 2007, astro$\mathrm{ph} / 0711.3014$

Kormendy, J., Kennicutt, R.C., Jr.: 2004, ARA\&A 42, 603

Kormendy, J., Fisher, D.B.: 2005, RMxAA 23, 101

Liske, J., Lemon, D.J., Driver, S.P., Cross, N.J.G., Couch, W.J.: 2003, MNRAS 344, 307

Mastropietro, C., Moore, B., Mayer, L., et al.: 2005, MNRAS 364, 607

Matthews, L.D., Gallagher, J.S.: 1997, AJ 114, 1899

Mitronova, S.N., Karachentsev, I.D., Karachentseva, V.E., Jarrett, T.H., Kudrya, Yu.N.: 2004, Bull. Special Astrophys. Obs. 57, 5

Ogorodnikov, K.F.: 1957, SvA 1, 748

Ogorodnikov, K.F.: 1958, SvA 2, 375

Okamoto, T., Eke, V.R., Frenk, C.S., Jenkins, A.: 2005, MNRAS 363,1299

Piontek, F., Steinmetz, M.: 2008, in preparation

Purcell, C.W., Kazantzidis, S., Bullock, J.S.: 2008, astro$\mathrm{ph} / 0810.2785$

Roediger, E., Hensler, G.: 2005, A\&A 433, 875

Scannapieco, C., Tissera, P.B., White, S.D.M., Springel, V.: 2008, MNRAS 389, 1137

Simard, L., Willmer, C.N.A., Vogt, N.P., et al.: 2002, ApJS 142, 1

Simard, L., Clowe, D.C., Desai, V., et al.: 2008, A\&A, submitted

Steinmetz, M.: 2003, Ap\&SS 284, 325

Stewart, K.R., Bullock, J.S., Wechsler, R.H., Maller, A.H., Zentner, A.R.: 2008, ApJ 683, 597

Toomre, A.: 1977, in: B.M. Tinsley, R.B. Larsen (eds.), Evolution of Galaxies and Stellar Populations, p. 402

Tran, K.-V.H., Moustakas, J., Gonzalez, A.H., Bai, L., Zaritsky, D., Kautsch, S. J.: 2008, ApJ 683, L17

Vincent, R.A., Ryden, B.S.: 2005, ApJ 623, 137

Vorontsov-Vel'yaminov, B.: 1967, in: M. Hack (ed.), Modern Astrophysics, p. 347

Vorontsov-Vel'yaminov, B.: 1974, SvA 17, 452

Walcher, C.J., Böker, T., Charlot, S., et al.: 2006, ApJ 649, 692

York, D.G., Adelman, J., Anderson, J.E., Jr., et al.: 2000, AJ 120, 1579 